\title{
Caracterização do perfil de jovens jogadores de futebol: uma análise das habilidades técnicas e variáveis antropométricas
}

\section{Profile characterization of young soccer players: an analysis of the technical skills and anthropometric variables}

Luiz Guilherme Cruz Gonçalves ${ }^{1 *}$, Rodrigo Leal de Queiroz Thomaz Aquino ${ }^{1}$, Hugo Tourinho Filho $^{2}$, Enrico Fuini Puggina²

ARTIGO ORIGINAL | ORIGINAL ARTICLE

\begin{abstract}
O objetivo deste estudo foi verificar as variáveis antropométricas, maturação biológica e as características técnicas de jovens futebolistas. 151 jogadores das categorias de base foram avaliados para estatura, massa corporal e dobras cutâneas, Pico de Velocidade de Crescimento (PVC) e testes específicos. Foram encontradas diferenças de sub-09 à sub-13 para \% de gordura, e sub-09 a sub-14 para estatura, massa corporal, massa gorda e passe à parede. O PVC foi diferente entre as categorias sub-09 a sub-16. Ademais, diferenças foram encontradas entre o sub-09 a sub-13 para condução de bola, e sub-09 a sub-15 no controle de bola. Conclui-se que a antropometria, a maturação biológica e a habilidade técnica podem ser influenciadas pelo crescimento e desenvolvimento inerentes à infância e adolescência.

Palavras-chaves: Formação esportiva; Esportes, Crescimento e Desenvolvimento.
\end{abstract}

ABSTRACT

The objective of this study was to verify the anthropometric variables, biological maturation and technical characteristics of young soccer players. 151 players in the lower grades were evaluated for height, body mass and skinfold, Growth Rate Peak (PVC) and specific tests. Differences in sub-09 to under- 13 for\% fat were found, and under-09 to under-14 to height, body mass, fat mass and pass the wall. PVC was different between the categories under-09 to under-16. In addition, there were differences between the sub-09 to under-13 for ball driving and sub-09 to U-15 in ball control. It is concluded that the anthropometry, maturation and technical skill may be influenced by the growth and development inherent in childhood and adolescence.

Keywords: Sports training; Sports, Growth and Development.

Artigo recebido a 01.04.2015; Aceite a 17.03.2016

${ }^{1}$ Programa de Pós-Graduação em Reabilitação e Desempenho Funcional, Faculdade de Medicina de Ribeirão Preto, USP, Ribeirão Preto, Brasil.

${ }^{2}$ Escola de Educação Física e Esporte de Ribeirão Preto, USP, Ribeirão Preto, Brasil.

* Autor correspondente: USP-RP, Av. Bandeirantes 3.900, Monte Alegre, 14040-900, Ribeirão Preto. E-mail: luiz.goncalves@usp.br 


\section{INTRODUÇÃo}

O futebol ocupa um lugar importante na cultura esportiva mundial entre crianças e jovens das mais diversas classes sociais e culturais. Muito provavelmente isso é resultado da sua popularidade entre os adultos e da sua universalidade da prática (Ramos, 2003). Fato evidenciado no Brasil com maior intensidade devido a aspectos como a grande miscigenação do seu povo e sua história vitoriosa no cenário mundial, transformando o futebol em uma identidade nacional.

Em relação a esse esporte, considera-se a imprevisibilidade e aleatoriedade das ações características predominantes em seu jogo. De modo que os aspectos físicos, táticos, psicológicos e técnicos se inter-relacionam e exercem influência mútua durante uma partida de futebol, tornando-o um sistema complexo (Guimarães \& Paoli, 2013).

Atrelada a essa complexidade, fatores como a necessidade elaborada da consciência corporal, direcional e espacial, sincronia, ritmo e sequência de movimentos (Gallahue, 2000) exigidos na execução das ações requeridas no jogo de futebol podem ser variáveis explicativas para a dificuldade de aprendizagem dos jovens jogadores de futebol, principalmente quando estão no processo de crescimento e desenvolvimento.

Associado a tais dificuldades na aprendizagem, verifica-se que a variação individual no processo de crescimento e desenvolvimento interfere na maturação biológica dos jovens. Desta maneira, o estado maturacional difere mesmo quando comparados indivíduos com a mesma idade cronológica (Villar \& Zuhl, 2006). De modo que o desempenho no futebol, assim como em outras modalidades esportivas, sofre influência da maturação e, portanto, observa-se a necessidade de investigação de como os jovens jogadores de futebol se desenvolvem ao longo dos anos.

A literatura tem investigado o desenvolvimento dos jovens jogadores por meio da análise de variáveis antropométricas, do estado de maturação e das habilidades motoras específicas do futebol. Malina e Bouchard (2004) acompanharam o comportamento das variáveis antropométricas em jovens atletas do sexo masculino, onde verificaram um aumento da massa corporal e estatura com o avanço da idade cronológica. Além de observarem que a massa adiposa (\% de gordura) permanece equilibrada durante a fase da adolescência. Em relação à maturação, verifica-se que o PVC máximo ocorre na idade média de 14 anos para os jogadores do sexo masculino (Mirwald, Baxter-Jones, Bailey, $\&$ Beunen, 2002).

No que refere-se ao desempenho técnico, autores têm analisado as habilidades motoras específicas do futebol por meio de testes específicos, como controle de bola, chute, condução de bola e passe (Federação Portuguesa de Futebol [FPF], 1986; Figueiredo, 2001; Kiekendall, Gruber, \& Johnson, 1987; Malina et al., 2005; Seabra, Maia, \& Garganta, 2001; Vaeyens et al., 2006; Valente-dos-Santos et al., 2012), verificando variabilidade nos resultados principalmente na idade entre 11 e 14 anos.

Apesar do Brasil ser reconhecido internacionalmente por apresentar jogadores de futebol com elevada capacidade técnica (Miranda, Antunes, Pauli, Puggina, \& da Silva, 2013), percebe-se escassez de estudos que investigam o comportamento dos aspectos técnicos com uma amostra nacional. Além disso, se faz necessário mais estudos sobre variáveis antropométricas, maturacionais e técnicas no decorrer das diferentes categorias de formação, verificando possíveis alterações e interações entre os fatores de crescimento, maturação e técnico.

Ademais, informações pertinentes ao processo de desenvolvimento antropométrico, maturacional e técnico podem contribuir para ampliação do conhecimento de maneira aplicada, ajudando treinadores e cientistas do esporte na obtenção de referenciais nesta temática ao longo das categorias de formação.

Com base no exposto, o objetivo do presente estudo foi avaliar e comparar as variáveis antropométricas, maturação biológica e as características técnicas de jovens jogadores brasileiros de futebol ao longo de diferentes faixas etárias. 


\section{MÉTODO}

\section{Participantes}

Foram estudados 151 atletas divididos em nove diferentes categorias, Sub-9 $(n=09)$, Sub$10(n=13)$, Sub-11 $(n=13)$, Sub-12 $(n=19)$, Sub-13 $(n=21)$, Sub-14 $(n=21)$, Sub-15 $(n=$ 14), Sub-16 $(n=18), \quad$ Sub-17 $\quad(n=24)$ pertencentes a uma escola de futebol de um clube profissional de futebol do interior do estado de São Paulo - Brasil, na qual disputavam competições a nível regional. Como critério de inclusão foi adotado a prática de no mínimo um ano ininterrupto de treinamento no futebol, para minimizar a variabilidade entre os participantes do estudo.

Este estudo foi aprovado pelo Comitê de Ética em Pesquisa do Hospital das Clínicas da Faculdade de Medicina de Ribeirão Preto da Universidade de São Paulo, sob parecer $\mathrm{n}^{\circ}$. 711.038. Todos os responsáveis pelos participantes assinaram um termo de consentimento livre e esclarecido informando os procedimentos e objetivos do estudo.

\section{Instrumentos e Procedimentos}

Antropometria

Foram realizadas medidas da estatura (m), massa corporal $(\mathrm{kg})$ e dobras cutâneas por meio dos seguintes procedimentos: estatura alongada através da utilização de um estadiômetro Sanny ${ }^{\circledR}$ com precisão de $1 \mathrm{~cm}$ e medida máxima de $2,20 \mathrm{~m}$ e massa corporal foi mensurada com uma balança digital com precisão de 100 g, modelo 2096 da marca Toledo ${ }^{\circledR}$.

\section{Composição Corporal}

Para realização da medida da gordura corporal, foram utilizados um adipômetro científico da marca Sanny ${ }^{\circledR}$ e o modelo antropométrico multicomponente para cálculo simultâneo da massa de gordura (MG) e \% de gordura de Machado (2009). As dobras cutâneas triciptal, supraíliaca, abdominal vertical, coxa medial e os perímetros de braço relaxado e coxa medial foram avaliados de forma rotacional ou sequencial, sendo a mediana de três avaliações utilizada para os cálculos.

\section{Maturação Biológica}

A maturação biológica foi determinada em anos pelo PVC proposta por Mirwald, BaxterJones, Bailey, e Beunen (2002). O estabelecimento da maturação foi mensurado por meio de uma fórmula específica para homens, na qual corresponde à distância, em anos, a que um indivíduo se encontra do PVC. O valor deve ser positivo (extrapolando o PVC) ou negativo (não superando o PVC).

Testes de habilidades motoras específicas do futebol

O protocolo de cada teste foi explicado previamente aos jovens jogadores de futebol avaliados. Os testes de habilidades motoras especificas do futebol foram baseados em dois autores Kiekendall, Gruber, e Johnson (1987) e FPF (1986): passa à parede, controle de bola, condução de bola e chute. Todos os testes foram realizados no mesmo local, dia e horário de treinamento dos jovens. Previamente ao início dos testes foi realizado um aquecimento padrão. Os testes foram realizados em formato de circuito, adotando a mesma sequência descrita acima, e também houve períodos de recuperação entre os testes.

O protocolo de operacionalização do teste passe à parede é composto por um desenho de um quadrado na parede, com $1.22 \mathrm{~m}$ de altura (a partir do solo) e $2.44 \mathrm{~m}$ de largura, e, no solo, foi determinada uma área à uma distância de $1.83 \mathrm{~m}$ da parede, demarcado com 4 cones em formato de retângulo de $4.23 \mathrm{~m}$ de comprimento por $1.83 \mathrm{~m}$ de largura. O jovem atleta foi posicionado dentro do retângulo, iniciando o teste através de um estímulo sonoro do avaliador. A prova consistia em realizar a máxima quantidade de toques na parede em 20 segundos. Porém, cada vez que o jovem errava a área alvo, era descontado um toque do total alcançado, o mesmo ocorreria quando o participante fazia o passe fora da área demarcada no solo ou quando pegava a bola com a mão. Foram realizadas três tentativas, sendo considerada a melhor pontuação (Kiekendall et al., 1987).

O processo de execução do teste de controle de bola é constituído por um espaço de 9 x $9 \mathrm{~m}$. Onde, posicionou-se o executante dentro do quadrado e com o estímulo sonoro do avaliador 
dado, abriu-se a contagem dos toques. Porém, a interrupção da contagem acontecia quando: a bola tocava ao solo ou executante saiu do respectivo quadrado. Foram adotadas duas tentativas e foi determinada a maior quantidade de toques como o melhor resultado (FPF, 1986).

$\mathrm{O}$ procedimento de realização do teste de condução de bola é formado a partir do posicionamento do executante ao lado do primeiro cone, logo, que o examinador deu o estímulo sonoro, o jovem jogador de futebol tinha o objetivo de conduzir a bola o mais rápido possível no percurso com o formato de um "M". Em um quadrado de $9 \times 9 \mathrm{~m}$ e um cone no meio do quadrado, caso o jogador derruba-se algum dos 5 cones, esse deveria parar para levantar e prosseguir o teste. Ao final, se o participante deixar algum cone fora da marca, ele seria eliminado. Foram realizadas duas tentativas, levando em considerando o menor tempo como o melhor resultado (FPF, 1986).

O formato de aplicação do teste do chute é feito com o início no posicionamento do jovem jogador de futebol com a bola a 9 metros das traves, tendo como objetivo chutar a bola para que a mesma atinja um dos alvos, de acordo com a pontuação respetiva: 1 ponto (espaço central), 2 pontos (espaço central superior), 3 pontos (retângulos laterais), 5 pontos (quadrados superiores laterais). Se a bola batesse na trave ou ia para fora do gol era contado como 0 ponto. $\mathrm{O}$ gol tinha as dimensões de $2 \mathrm{~m}$ de altura por $3 \mathrm{~m}$ de largura. Se a bola alcançasse-se a fita que dividia os espaços, seria considerada a maior área de pontuação. Buscou-se obter a maior pontuação, considerando a soma de cinco tentativas (FPF, 1986).

\section{Análise estatística}

Os resultados foram descritos em média e desvio padrão e tratados utilizando-se dos pacotes estatísticos Graph-Pad Instat ${ }^{\circledast}$ e Prism $^{\circledR}$ (La Jolla, EUA). Para caracterização da amostra comparação entre as médias da antropometria, composição corporal, maturação biológica e os testes das habilidades motoras especificas do futebol entre as categorias, determinando as diferenças encontradas entre as faixas etárias. A distribuição normal dos dados foi verificada pelo teste de Shapiro-Wilk. Foi utilizado o teste de one-way anova para possíveis diferenças, seguido, quando necessário, do pós-teste de Tukey-Kramer. O nível de significância utilizado foi de $5 \%$.

\section{RESULTADOS}

Os resultados antropométricos, composição corporal e maturação biológica foram comparados para verificar a existência de diferenças entre as categorias que compuseram este estudo.

Tabela 1

Média e desvio padrão dos valores antropométricos, composição corporal e maturação biológica referente a cada categoria.

\begin{tabular}{lccccccccc}
\hline \multicolumn{1}{c}{ Variáveis } & Sub-9 & Sub-10 & Sub-11 & Sub-12 & Sub-13 & Sub-14 & Sub-15 & Sub-16 & Sub-17 \\
\hline Idade Cron. (anos) & $8.8 \pm 0.6$ & $9.6 \pm 0.5$ & $10.7 \pm 0.5$ & $11.7 \pm 0.4$ & $12.6 \pm 0.5$ & $13.9 \pm 0.3$ & $14.5 \pm 0.5$ & $15.7 \pm 0.5$ & $16.8 \pm 0.4$ \\
Estatura (cm) & $130.2 \pm 5.8^{\mathrm{a}}$ & $139.7 \pm 4.9^{\mathrm{b}}$ & $142.3 \pm 4.5^{\mathrm{c}}$ & $148.4 \pm 6.0^{\mathrm{d}}$ & $155.3 \pm 6.5^{\mathrm{e}}$ & $163.1 \pm 8.5^{\mathrm{f}}$ & $171.7 \pm 5.4$ & $173.7 \pm 5.1$ & $175.3 \pm 7.8$ \\
Massa Corp. (Kg) & $30.5 \pm 7.6^{\mathrm{a}}$ & $35.6 \pm 7.2^{\mathrm{b}}$ & $33.9 \pm 4.5^{\mathrm{c}}$ & $41.2 \pm 6.3^{\mathrm{d}}$ & $50.9 \pm 11.3^{\mathrm{e}}$ & $52.4 \pm 11.8^{\mathrm{f}}$ & $57.0 \pm 7.9$ & $60.8 \pm 6.9$ & $63.1 \pm 7.6$ \\
PVC (anos) & $-3.9 \pm 0.5^{\mathrm{a}}$ & $-3.3 \pm 0.3^{\mathrm{b}}$ & $-3.0 \pm 0.4^{\mathrm{c}}$ & $-2.5 \pm 0.4^{\mathrm{d}}$ & $-2.0 \pm 0.4^{\mathrm{e}}$ & $-0.3 \pm 0.9^{\mathrm{f}}$ & $0.6 \pm 0.5^{\mathrm{g}}$ & $1.5 \pm 0.4^{\mathrm{h}}$ & $2.2 \pm 0.5$ \\
Massa Gorda (Kg) & $5.6 \pm 4.2^{\mathrm{a}}$ & $7.8 \pm 5.7$ & $3.9 \pm 2.0^{\mathrm{b}}$ & $7.7 \pm 4.2$ & $11.9 \pm 5.7^{\mathrm{c}}$ & $8.0 \pm 5.8$ & $6.9 \pm 3.1$ & $6.7 \pm 2.6$ & $8.9 \pm 3.5$ \\
\% de Gordura & $16.6 \pm 8.6$ & $19.8 \pm 11.9^{\mathrm{a}}$ & $11.0 \pm 4.3^{\mathrm{b}}$ & $17.6 \pm 8.1$ & $22.4 \pm 7.9^{\mathrm{c}}$ & $13.9 \pm 7.9$ & $11.6 \pm 3.9$ & $10.7 \pm 3.2$ & $13.9 \pm 4.2$ \\
\hline
\end{tabular}

Estatura $={ }^{\text {a }}$ sub-9 $\mathrm{x}$ sub10, sub-11, sub-12, sub-13, sub-14, sub-15, sub-16, sub-17; ${ }^{\text {b }}$ sub-10 x sub-12, sub-13, sub-14, sub-15, sub-16, sub-17; ${ }^{\mathrm{c}}$ sub-11 x sub-13, sub-14, sub-15, sub-16, sub-17; ${ }^{\mathrm{d}}$ sub-12 $\mathrm{x}$ sub-13, sub-14, sub-15, sub-16, sub-17; ${ }^{\mathrm{e}}$ sub-13 x sub-14, sub-15, sub-16, sub-17; f sub-14 x sub-15, sub-16, sub-17.

Massa Corp. $={ }^{\mathrm{a}}$ sub-9 x sub-13, sub-14, sub-15, sub-16, sub-17; ${ }^{\mathrm{b}}$ sub-10 x sub-13, sub-15, sub-16, sub-17; ${ }^{\mathrm{c}}$ sub-11 x sub-13, sub-14, sub-15, sub-16, sub-17; ${ }^{\mathrm{d}}$ sub-12 x sub-13, sub-14, sub-15, sub-16, sub-17; ${ }^{\mathrm{e}}$ sub-13 x sub-16, sub-17; ${ }^{\mathrm{f}}$ sub-14 x sub-17. PVC $={ }^{a}$ sub-9 $\times$ sub-11, sub-12, sub-13, sub-14, sub-15, sub-16, sub-17; ${ }^{\mathrm{b}}$ sub-10 x sub-12, sub-13, sub-14, sub-15, sub-16, sub-17; ' ${ }^{\mathrm{c}}$ sub-11 x sub-12, sub-13, sub-14, sub-15, sub-16, sub-17; ${ }^{\mathrm{d}}$ sub-12 x sub-13, sub-14, sub-15, sub-16, sub-17; ${ }^{\mathrm{e}}$ sub-13 x sub-14, sub-15, sub-16, sub-17; ${ }^{\mathrm{f}}$ sub-14 x sub-15, sub-16, sub-17; ${ }^{\mathrm{g}}$ sub-15 x sub-16, sub-17; ${ }^{\mathrm{h}}$ sub-16 $\mathrm{x}$ sub-17.

Massa Gorda $={ }^{\text {a }}$ sub-9 $\times$ sub-13; ${ }^{\text {b }}$ sub-11 $\times$ sub-13, sub-17; ${ }^{\mathrm{c}}$ sub-13 $\mathrm{x}$ sub-15, sub- 16 .

$\%$ de Gordura $={ }^{a}$ sub-10 $\times$ sub- 16 ; ${ }^{b}$ sub- $11 \times$ sub-13; ${ }^{\mathrm{c}}$ sub- $13 \times$ sub-14, sub- 15 , sub- 17 . 
A Tabela 1 está evidenciando a descrição e comparação dos valores, representados por média e desvio padrão, entre todas as categorias. Foram analisadas seis variáveis, sendo relacionadas à idade cronológica, antropometria, composição corporal e maturação biológica.

Em geral, as medidas de estatura e massa corporal aumentam de forma progressiva e contínua à medida que os jovens atletas se tornam mais velhos, especificamente, da categoria sub-9 até a categoria sub-14. Por outro lado, aos 15 anos há um platô das medidas dessas variáveis antropométricas, portanto, não se pode dizer que existe elevação significativa desde a primeira categoria até a última investigada nesse estudo.
Em relação a variável PVC, obteve-se diferença significante entre o sub-9 ao sub-16, percebendo que as mudanças da maturação biológica ocorrem até os 16 anos, e acima dessa idade, os valores se tornam positivos, ocorrendo à estabilização desta variável até a última categoria (sub-17).

Percebe-se que a dinâmica das variáveis massa gorda e $\%$ de gordura segue aumentando desde a categoria sub-9 até o sub-13 e, após o sub-14 há uma diminuição e manutenção dos resultados até a categoria sub-17, ou seja, a medida que os jovens ficam mais velhos até os 14 anos, eles ficam com maior massa gorda, porém esse quadro se inverte depois dessa idade, os mesmos emagrecem e permanecem com os mesmos valores das variáveis massa gorda e \% de gordura.

Tabela 2

Média e desvio padrão dos resultados dos testes das habilidades motoras específicas do futebol referente cada categoria.

\begin{tabular}{lccccccccc}
\hline \multicolumn{1}{c}{ THMEF* } & Sub-9 & Sub-10 & Sub-11 & Sub-12 & Sub-13 & Sub-14 & Sub-15 & Sub-16 & Sub-17 \\
\hline Passe à Parede & 10.66 & 10.92 & 12.69 & 12.42 & 13.42 & 14.18 & 15.14 & 15.44 & 17.51 \\
(passes) & \pm & \pm & \pm & \pm & \pm & \pm & \pm & \pm & \pm \\
& 2.34 & 2.21 & 2.62 & 2.73 & 2.58 & 1.72 & 2.90 & 2.22 & 2.39 \\
Controle de Bola & 4.55 & 8.53 & 18.92 & 20.42 & 34.71 & 36.23 & 40.71 & 109.72 & 89.66 \\
(toques) & \pm & \pm & \pm & \pm & \pm & \pm & \pm & \pm & \pm \\
& 1.23 & 7.53 & 14.86 & 36.32 & 39.36 & 37.29 & 33.87 & 103.66 & 82.51 \\
Condução de Bola & 17.54 & 17.77 & 15.19 & 15.12 & 15.68 & 14.27 & 13.39 & 13.10 & 13.07 \\
(seg.) & \pm & \pm & \pm & \pm & \pm & \pm & \pm & \pm & \pm \\
& 1.65 & 1.54 & 1.31 & 1.42 & 2.69 & 0.98 & 0.98 & 0.73 & 1.09 \\
Chute (pontos) & 3.42 & 3.38 & 6.61 & 5.36 & 5.33 & 5.92 & 5.74 & 5.55 & 7.15 \\
& \pm & \pm & \pm & \pm & \pm & \pm & \pm & \pm & \pm \\
\hline
\end{tabular}

*Testes das Habilidades Motoras Específicas do Futebol.

Os dados coletados a partir dos testes específicos para as habilidades técnicas foram realizados em todos os jovens jogadores de futebol nas nove diferentes categorias: sub-9, sub-10, sub-11, sub-12, sub-13, sub-14, sub-15, sub-16 e sub-17. A Tabela 2 mostra os dados descritivos, representados por média e desvio padrão.

Os resultados dos testes das habilidades motoras específicas do futebol foram comparados para verificar a existência de diferenças entre todas as categorias (Figura 2).

Pode-se observar adaptações e melhoras no desempenho do teste de passe à parede (Figura 1 - A) entre as categorias sub-9 à sub-14. Uma vez que, após esse período chave de aprendizagem da capacidade técnica em especial (passe) acontece à manutenção dos resultados até a categoria sub17.

A mudança nos resultados frente à melhora do desempenho no teste de controle de bola ocorre do sub-9 até o sub-15 (Figura 1 - B). Um período em que pode-se verificar que há melhora dos dados de forma gradativa até o sub-15 e, logo nas categorias seguintes parece haver a manutenção do rendimento nessa habilidade técnica.

Novamente, o período em que acontece a melhora dos resultados do teste de condução de bola (Figura $1-\mathrm{C}$ ) é parecido com os demais testes, isto é, o momento em que ocorre aperfeiçoamento do desempenho na condução de bola é entre sub-9 e o sub-13, acontecendo uma redução do tempo dos resultados de forma progressiva até os 13 anos. Desta maneira, no 
sub-14 até a o sub-17 parece não existir evolução no rendimento técnico, podendo observar que o desempenho do sub-14 e sub-17 são semelhantes.
No que se refere aos valores médios do teste de chute (Figura $1-D$ ), não foram encontradas diferenças significantes quando comparadas às diferentes categorias.
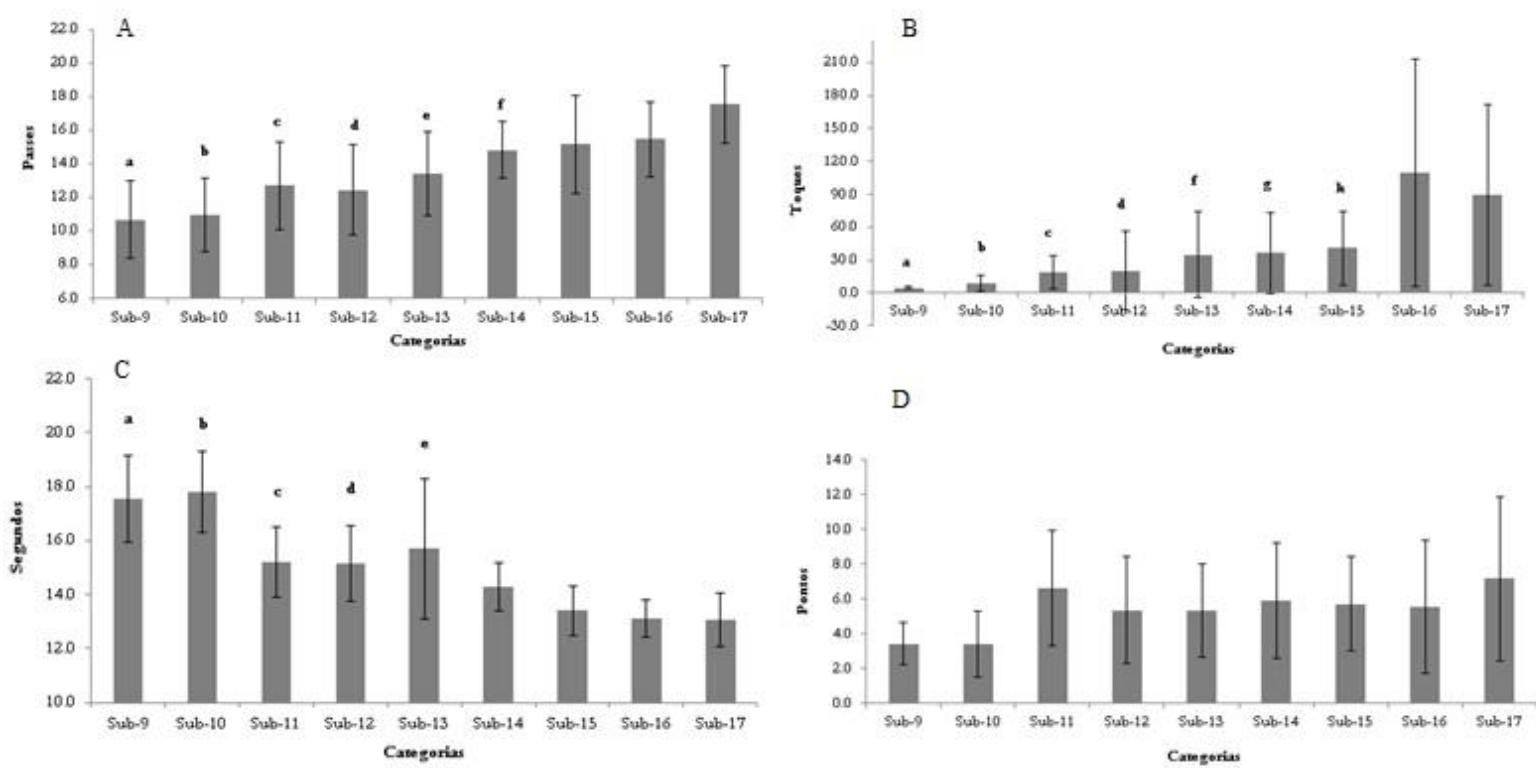

D

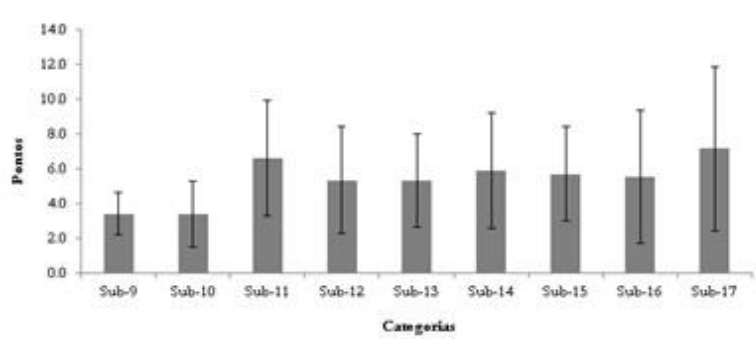

Figura 1. Valores médios do teste de passe à parede (passes) - A; controle de bola (toques) - B; condução de bola (segundos) - C e teste de chute (pontos) - D nas diferentes categorias estudadas. A: a sub- 9 x sub-14 $-\mathrm{p}<0,01$, sub-15, sub-16, sub-17 - $\mathrm{p}<0,001$; ${ }^{\mathrm{b}}$ sub-10 $\mathrm{x}$ sub-14, sub-15, sub-16, sub-17 $-\mathrm{p}<0,001$; $^{\mathrm{c}}$ sub-11 x sub-17 $-\mathrm{p}<0,001$; $^{\mathrm{d}}$ sub-12

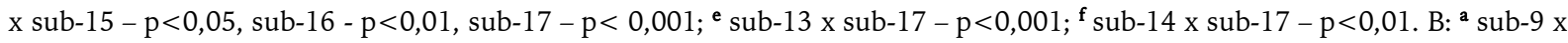
sub-16 - $p<0,001$, sub-17 - $p<0,01$; $^{\mathrm{b}}$ sub-10 x sub-16 - $\mathrm{p}<0,001$, sub-17 $-\mathrm{p}<0,001$; $^{\mathrm{c}}$ sub-11 x sub-16 - $\mathrm{p}<0,001$, sub-17 -

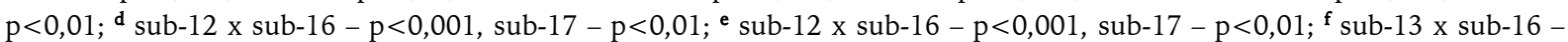
$\mathrm{p}<0,01$, sub-17 - $\mathrm{p}<0,05$; $^{\mathrm{g}}$ sub-14 $\mathrm{x}$ sub- $16-\mathrm{p}<0,01$, sub- $17-\mathrm{p}<0,05$; $^{\mathrm{h}}$ sub-15 x sub- $16-\mathrm{p}<0,05$. C: ${ }^{\mathrm{a}}$ sub-9 $\mathrm{x}$ sub-11 $\mathrm{p}<0,005$, sub-12 $-\mathrm{p}<0,01$, sub-14, sub-15, sub-16, sub-17 $-\mathrm{p}<0,001$; $^{\mathrm{b}}$ sub-10 $\mathrm{x}$ sub-11, sub-12 $-\mathrm{p}<0,001$, sub-13 $-\mathrm{p}<0,01$, sub-14, sub-15, sub-16, sub-17 - $\mathrm{p}<0,01$; $^{\mathrm{c}}$ sub-11 x sub-15 - $\mathrm{p}<0,05$, sub- 16 - $\mathrm{p}<0,01$, sub-17 $-\mathrm{p}<0,001$; ${ }^{\mathrm{d}}$ sub-12 x sub-15 $\mathrm{p}<0,05$, sub-16 $-\mathrm{p}<0,01$, sub- $17-\mathrm{p}<0,001$; ${ }^{\mathbf{e}}$ sub- $13 \times$ sub-15, sub- 16 , sub- $17-\mathrm{p}<0,001$.

\section{DISCUSSÃO}

O principal achado desse estudo foi com relação às análises dos testes para as habilidades motoras específicas do futebol na qual pode-se verificar que há uma evolução na medida que se vai progredindo na categoria até, aproximadamente os 14 anos, já que, após esta categoria é possível observar uma estabilização do desempenho técnico até os 17 anos. Especificamente, para o teste de passe à parede a melhora dos resultados acontece de forma progressiva até os 14 anos, já para o teste de controle de bola os ganhos verificam-se até o sub15 e, para o teste de condução de bola os incrementos dos valores ocorrem até os 13 anos, entretanto, o teste de chute é a única exceção quando comparado aos demais testes, ou seja, o teste de chute não segue uma dinâmica de melhora do desempenho com o aumento da idade cronológica.

Uma limitação prática vista sobre esse teste do chute foi o fato de que os jovens atletas com níveis positivos de PVC, acima da categoria sub15, buscavam os alvos com pontuações mais elevadas, porém o que acontecia era que esses apresentavam os maiores índices de erro, como chutes para fora do gol, obtendo pontuação igual à zero. Já os jovens atletas com níveis negativos de PVC, abaixo da categoria sub-15, buscavam alvos teoricamente mais fáceis, obtendo uma pontuação não tão alta, porém uniforme. Contudo, pode-se discutir sobre a característica estrutural deste teste na avaliação do chute.

Os resultados obtidos nos testes das habilidades motoras específicas do futebol podem ser explicados por um conjunto de fatores que influenciam no desenvolvimento do 
aprendizado técnico da modalidade em questão, sendo eles: a idade cronológica, a maturação biológica, desempenho atlético e as variáveis antropométricas (Dias, 2007).

Os dados deste estudo corroboram com os estudos de Abreu (2008) e Dias (2007) onde indicam que, o desenvolvimento do processo de formação e preparação esportiva aliado ao avanço da idade cronológica, favorece a melhora dos resultados dos testes de habilidade motora no futebol.

Também foi possível detectar essa concordância no estudo de Malina, Ribeiro, Aroso, e Cumming (2007) com jovens jogadores de futebol dos 13 aos 15 anos, onde foi identificado que o estado maturacional e a resistência aeróbia contribuem de forma positiva sobre a pontuação e o tempo nos testes das habilidades motoras específicas do futebol. Por outro lado, a estatura apresenta contribuição negativa para o desempenho técnico. Ou seja, estas três variáveis juntas são responsáveis por $29 \%$ de variação nos resultados da capacidade técnica.

O estudo de Valente-dos-Santos et al. (2012) corrobora com os resultados encontrados neste estudo, onde os autores analisaram o perfil de jovens jogadores de futebol por meio da avaliação da idade cronológica, antropometria, maturação e dos testes das habilidades motoras específicas do futebol em diferentes categorias, verificando que os valores do desempenho técnico melhoram com o avanço da idade cronológica e do estado maturacional.

No presente estudo, de maneira geral, observou-se variabilidade nos resultados das habilidades motoras específicas do futebol, com exceção ao teste de chute, entre as idades de 11 a 14 anos. Esse fato associa-se as mudanças no estado de maturação marcadas por essa fase. $\mathrm{O}$ mesmo não acontece nas categorias acima de 14 anos, grupos esses pertencentes quase que exclusivamente por indivíduos pós-púberes (Dias, 2007). Além disso, pode-se justificar tal variabilidade por aspectos individuais, como o talento esportivo e a aptidão motora para a modalidade (Williams \& Reilly, 2000).

Por outro lado, segundo Horta (2003) o estado de maturação parece ser independente da habilidade técnica no futebol, ou seja, o indivíduo pós-púbere não necessariamente apresenta melhores resultados nos testes das habilidades motoras específicas do futebol em relação aos indivíduos pré-púberes e púberes, o que não corrobora com os dados do presente estudo.

Além dos fatores citados, existem uma série de elementos que influenciam no desenvolvimento das habilidades motoras do futebol durante a formação dos esportistas, como o conteúdo do treinamento, a oportunidade da prática deliberada/especializada e $\mathrm{o}$ seu respectivo tempo, perfil dos atletas, motivação, marcadores genéticos, aparecimento de lesões, além das mudanças biopsicossociais que estas crianças e adolescentes estão passando durante essa fase da vida (Morris, 2000; Williams \& Franks, 1998; Williams \& Reilly, 2000).

Além disso, outros elementos podem explicar as mudanças no desempenho técnico que ocorrem ao longo das categorias e até mesmo dentro das próprias categorias, tais como: as diferenças individuais no tempo e ritmo de maturação do controle neural e nos processos perceptivo-cognitivos (pe. antecipação e estratégias de busca visual) (Morris, 2000).

No tocante do processo de desenvolvimento das habilidades técnicas ao longo das categorias de formação, Martin (1982) verificou que o período entre 6 aos 14 anos parece ser o mais sensível para o desenvolvimento do gesto motor, pois ocorre uma grande evolução na coordenação e controle motor. Ademais, entre 10 e 14 anos o principal foco no treinamento deve estar na continuidade da aprendizagem e no aperfeiçoamento das habilidades técnicas (Barbanti, 2001; Martin, 1982). Por consequência, esse é o período ideal para desenvolver as habilidades motoras complexas (FPF, 1986; Gallahue \& Ozmun, 1995; Weineck, 1999) próximas às exigências da modalidade esportiva em questão.

Logo, observou-se nos resultados deste estudo um panorama de evolução no desempenho técnico entre as faixas etárias dos 9 aos 14 anos, o que corrobora com o período sensível supracitado no estado de Martin (1982).

Em relação à estatura e a massa corporal, o presente estudo encontrou resultados 
semelhantes aos encontrados na literatura específica desta temática (Abreu, 2008; Dias, 2007; Moore, Moore, Klentrou, Sullivan, \& Falk, 2010; Neves, 2010; Valente-dos-Santos et al., 2012; Vandendriessche et al., 2012; Vänttinen, Blomqvist, Nyman, \& Häkkinen, 2011). As medidas antropométricas parecem ser semelhantes, independente da nacionalidade. $\mathrm{O}$ aumento da estatura e da massa corporal são processos normais durante o crescimento e desenvolvimento humano.

Além de que, o aumento da massa corporal tende a seguir a mesma amplitude da curva do aumento em estatura. Assim, esse acréscimo da massa corporal é motivado pelo incremento da estatura e também da massa muscular (Malina \& Bouchard, 2004).

Peña Reyes e Malina (2004) ainda sugerem que a massa corporal aumenta de acordo com o avanço do estado maturacional, ou seja, à medida que o jovem pré-púbere passa a ser púbere e póspúbere.

Quanto à maturação biológica, no estudo de Neves (2010) verificou-se que os resultados médios dos jovens jogadores de futebol portugueses com idades de 13-14 anos estavam a -1,37 anos do PVC. Por outro lado, Abreu (2008) mostra média referente à maturação biológica de, também, jovens jogadores de futebol portugueses que apresentavam faixa etária entre 13 e 14 anos estavam a $-0,35$ anos do PVC. Desse jeito, o estudo de Abreu (2008) corrobora com os achados deste estudo em contrapartida do estudo de Neves (2010).

De maneira sumarizada, a literatura e os resultados encontrados no presente estudo mostram que nas idades abaixo dos 14 anos há valores negativos e acima dos 14 anos observa-se valores positivos para o PVC, ou seja, conforme aumenta a idade cronológica, aumentam também os valores do PVC. Nesse sentido, na idade média de 14 anos para os sujeitos do sexo masculino normalmente ocorre o PVC (Mirwald et al., 2002).

No que diz respeito às variáveis de massa gorda e \% de gordura, no estudo de Valente-dosSantos et al. (2012) investigaram as diferentes características de jovens jogadores de futebol portugueses de sete diferentes faixas etárias, dos
11 até os 17 anos. Os resultados referentes à massa livre de gordura ou massa gorda foram semelhantes em alguns pontos e divergentes em outros. Na idade de 11 anos os resultados foram semelhantes, já nas idades de 12 e 13 anos verificou-se que os valores estavam abaixo do que foram apresentados neste estudo. Dos 14 até os 17 anos houve um aumento no estudo de Valente-dos-Santos et al. (2012), por outro lado, neste estudo, ocorreu um decréscimo.

No estudo de Vänttinen, Blomqvist, Nyman, e Häkkinen (2011) observou-se que os resultados referentes à \% de gordura são inferiores em todas as categorias quando comparado com os encontrados no presente estudo. Entretanto, foi verificado que há um aumento dos valores da \% de gordura entre a idade de 11 até os 14 anos e logo em seguida há um decréscimo dos valores até a idade de 17 anos, como foi visto nos resultados deste estudo.

Nikolaidis \& Karydis (2011) analisou a composição corporal que ocorre na adolescência, a amostra foi constituída de adolescentes gregos, todos pertenciam a clubes de futebol, e foram classificados em nove grupos de idade, ou seja, desde o sub-12 até o sub-21, entretanto, para discussão com os resultados do presente estudo, utilizou-se apenas os valores respectivos até a categoria sub-17. Os resultados obtidos na massa gorda foram semelhantes aos encontrados no presente estudo em relação a categoria sub- 12 . Quando analisados os valores das categorias sub13 à sub-17, obteve-se médias superiores a que foram constatados neste estudo. Já em relação a $\%$ de gordura se observou mudanças de acordo com a faixa etária, ou seja, há variabilidade entre a comparação dos dados de cada categoria.

Diante dos resultados encontrados, percebese que há um aumento da massa gorda e da \% de gordura até os 13 anos e diminuição até os 15 anos, quase estabilizando os valores até a última idade (17 anos). Esse processo pode ser elucidado pela repleção energética, onde ocorre o armazenamento da gordura para que logo em seguida seja utilizada na fase do pico de estirão do crescimento (Lacerda \& Accioly, 2003).

Os resultados deste estudo mostraram que a massa gorda foi reduzida e praticamente estabilizada após os 13 anos, resultado que pode 
ser explicado pela possibilidade do rápido aumento da estatura e pelo incremento dos níveis de secreção de testosterona, ao mesmo tempo em que pudesse ter aumentado a massa magra, por consequência, havendo aumento da massa corporal. Uma das características da testosterona é favorecer a diminuição da síntese de triglicerídeos (Arslanian \& Suprasongsin, 1997).

Portanto, existem diversos fatores, sejam eles do tipo tático, técnico, físico/fisiológico e psicológico que implicam na identificação, seleção e promoção de talentos esportivos. Às vezes, profissionais cometem o erro no momento da seleção do talento chamar-se a atenção apenas para o aspecto maturacional e não realmente para as demais capacidades, por exemplo, técnicotáticas (Malina, 2003).

Nesse sentindo, visto a natureza complexa, hierárquica, adaptativa e dinâmica a qual caracteriza-se o futebol, entende-se que os jogadores devem possuir uma formação polivalente, interligando os aspectos táticostécnicos, físicos-fisiológicos e psicológicos (Paoli, Silva, \& Soares, 2013).

Com isso, o desenvolvimento do talento é dependente de multifatores relacionados com o processo esportivo, porém, é de extrema valia o acompanhamento de um treinamento a longo prazo de forma sistemática, orientada e pedagógica (Weineck, 1999).

Assim, o talento mesmo possuindo características/desempenho acima da média comprovada para aquele grupo de atletas observados, ainda se faz necessário o processo de desenvolvimento para que o talento se torne uma comprovação da expectativa que fora criada no momento da seleção, pois sabe-se que o talento é uma parte limita do desenvolvimento do aprendizado de uma habilidade, uma vez que o nível de desempenho está intimamente ligada com o tempo e a qualidade do treinamento, ou seja, muitos anos de treino são necessários para alcançar o alto rendimento (Ericsson, Krampe, \& Tesch-romer, 1993).

Nessa perspectiva, o objetivo dos clubes em ter categorias de formação é proporcionar aos seus jovens atletas um momento de aperfeiçoamento em todas as capacidades. Entender e desenvolver diretrizes a serem seguidas por seus atletas dentro do clube. Por meio do ensino-aprendizagem-treinamento dos gestos motores, estimulação e conscientização tática, desenvolvimento da condição física e transparecendo uma conduta ética/educacional/moral alinhada com a filosofia do clube. Portanto, os treinadores deveram equilibrar os conteúdos do treinamento dentro e fora de campo para possibilitar o progresso de maneira harmônica de todas as capacidades (Williams \& Reilly, 2000).

\section{CONCLUSÕES}

Com base na análise dos dados, pode-se concluir que há evidências de que ocorrem mudanças antropométricas e evolução na capacidade técnica ao longo da adolescência, por isso, a exigência de entendimento desse assunto por parte dos profissionais do esporte é de extrema relevância na hora de transmitir suas instruções aos seus atletas a partir do conhecimento teórico na aplicação prática sobre as mudanças que norteiam durante essa fase da vida, principalmente em relação às questões de crescimento, desenvolvimento e estado de maturação. A fim de melhorar as interações entre o jovem atleta e o seu treinador e aperfeiçoar o processo de formação esportiva.

Por fim, considera-se fundamental a investigação relativa às crianças e jovens esportistas, cujo objetivo esteja no entendimento do comportamento multifatorial que envolve a prática esportiva.

\footnotetext{
Agradecimentos:

Escola Oficial de Futebol Botafogo Futebol Clube, Ribeirão Preto, Brasil.
}

\section{Conflito de Interesses:}

Nada a declarar.

Financiamento:

Nada a declarar

\section{REFERÊNCIAS}

Abreu, A. F. R. (2008). Selecção desportiva de jovens futebolistas. Estudo com a selecção sub-14 da Associação de Futebol de Coimbra (Dissertação de Mestrado em Treino Desportivo para Crianças 
36 | LGC Gonçalves, RLQ Thomaz-Aquino, H Tourinho-Filho, EF Puggina

e Jovens). Faculdade de Ciências do Desporto e Educação Física, Universidade de Coimbra, Coimbra.

Arslanian, S., \& Suprasongsin, C. (1997). Testosterone treatment in adolescents with delayed puberty: changes in body composition, protein, fat, and glucose metabolism. The Journal of Clinical Endocrinology and Metabolism, 82(10), 32133220. http://doi.org/10.1210/jcem.82.10.4293

Barbanti, V. J. (2001). Treinamento físico: bases científicas (3. ${ }^{a}$ ed.). São Paulo: CLR Balieiro.

Dias, N. M. M. (2007). Maturação sexual, morfologia e aptidão desportivo-motora em jovens futebolistas do escalão de infantis. Validade concorrente entre a pilosidade púbica autopercepcionada e avaliada por um perito (Dissertação de Mestrado em Treino Desportivo para Crianças e Jovens). Faculdade de Ciências do Desporto e Educação Física, Universidade de Coimbra, Coimbra.

Ericsson, K. A., Krampe, R. T., \& Tesch-romer, C. (1993). The role of deliberate practice in the acquisition of expert performance. Psychological Review, 100(3), 363-406. http://doi.org/10.1037/0033-295X.100.3.363

Federação Portuguesa de Futebol. (1986). Habilidades e destrezas do futebol: os skills do futebol. Lisboa: Federação Portuguesa de Futebol.

Figueiredo, A. (2001). Efeitos da selecção dimensional e funcional em jogadores de futebol infantis $e$ iniciados, segundo o tempo de permanência no escalão (Dissertação de Mestrado em Ciências da Actividade Física (Biocinética)). Faculdade de Ciências do Desporto e Educação Física, Universidade de Coimbra, Coimbra.

Gallahue, D. L. (2000). Educação Física desenvolvimentista. Cinergis, 1(1), 7-17.

Gallahue, D. L., \& Ozmun, J. C. (1995). Understanding motor development: infants, children, adolescents, adults (3. ${ }^{a}$ ed.). Iwoa: Benchmark.

Guimarães, M. B., \& Paoli, P. B. (2013). O treinamento técnico por posição no futebol: as especificidades na percepção dos técnicos de categorias de base do futebol mineiro. Revista Brasileira de Futebol, 4(1), 42-53.

Horta, L. (2003). Factores de predição do rendimento desportivo em atletas juvenis de futebol (Tese de Doutoramento). Faculdade de Medicina, Universidade do Porto, Porto.

Kiekendall, D., Gruber, J., \& Johnson, R. (1987). Measurement and evaluation for physical educators (2. ${ }^{a}$ ed.). Champaign Ilinois: Human Kinetics.

Lacerda, E. M. A., \& Accioly, E. (2003). Alimentação do pré-escolar e escolar. Em E. Accioly, C. Saunnders, \& E. M. A. Lacerda (Eds.), Nutrição em obstetrícia e pediatria (pp. 369-382). São Paulo: Cultura Médica.

Machado, D. R. L. (2009). Análise multivariada da composição corporal em jovens esportistas e não esportistas (Tese de Doutoramento em Educação
Física). Escola de Educação Física e Esporte, Universidade de São Paulo, São Paulo.

Malina, R. M. (2003). Growth and maturity status of young soccer (football) players. Em T. Reilly \& M. Williams (Eds.), Science and Soccer (pp. 287306). London: Routledge.

Malina, R. M., \& Bouchard, C. (2004). Growth, maturation and physical activity (2. ${ }^{\mathrm{a}}$ ed.). Champagn, IL: Human Kinetics.

Malina, R. M., Cumming, S. P., Kontos, A. P., Eisenmann, J. C., Ribeiro, B., \& Aroso, J. (2005). Maturity-associated variation in sport-specific skills of youth soccer players aged 13-15 years. Journal of Sports Sciences, 23(5), 515-522. http://doi.org/10.1080/0264041041000172992 8

Malina, R. M., Ribeiro, B., Aroso, J., \& Cumming, S. P. (2007). Characteristics of youth soccer players aged 13-15 years classified by skill level. British Journal of Sports Medicine, 41(5), 290-295. http://doi.org/10.1136/bjsm.2006.031294

Martin, D. (1982). Die leistungsfähigkeit und entwicklung derkinder als grundlage für den sportlichen leistungsaufbau. Beiheft $z u$ Leistungssport, 8(2), 47-64.

Miranda, R. E. E. P. C., Antunes, H. K. M., Pauli, J. R., Puggina, E. F., \& da Silva, A. S. R. (2013). Effects of 10-week soccer training program on anthropometric, psychological, technical skills and specific performance parameters in youth soccer players. Science \& Sports, 28(2), 81-87. http://doi.org/10.1016/j.scispo.2012.02.005

Mirwald, R. L., Baxter-Jones, A. D. G., Bailey, D. A., \& Beunen, G. P. (2002). An assessment of maturity from anthropometric measurements. Medicine and Science in Sports and Exercise, 34(4), 689694.

Moore, S. A., Moore, M., Klentrou, P., Sullivan, P., \& Falk, B. (2010). Maturity status in male child and adolescent athletes. The Journal of Sports Medicine and Physical Fitness, 50(4), 486-493.

Morris, T. (2000). Psychological characteristics and talent identification in soccer. Journal of Sports Sciences, $\quad 18(9), \quad 715-726$. http://doi.org/10.1080/02640410050120096

Neves, J. M. P. (2010). Caracterização multidimensional de jogadores de futebol com 13-14 anos (Dissertação de Mestrado em Treino Desportivo para Crianças e Jovens). Faculdade de Ciências do Desporto e Educação Física, Universidade de Coimbra, Coimbra.

Nikolaidis, P. T., \& Karydis, N. V. (2011). Physique and body composition in soccer players across adolescence. Asian Journal of Sports Medicine, 2(2), 75-82.

Paoli, P. B., Silva, C. D., \& Soares, A. J. G. (2013). Tendência atual da detecção, seleção e formação de talentos no futebol brasileiro. Revista Brasileira de Futebol, 1(2), 38-52.

Peña Reyes, M., \& Malina, R. M. (2004). Growth and maturity profile of youth swimmers in México. Em M. Coelho \& R. M. Silva (Eds.), Children and 
Youth in Organized Sports. Coimbra: Imprensa da Universidade.

Ramos, F. (2003). Futebol. Da "Rua» à Competição (2. ${ }^{a}$ ed.). Lisboa: Centro de estudos e Formação Desportiva.

Seabra, A., Maia, J., \& Garganta, J. (2001). Crescimento, maturação, aptidão física, força explosiva e habilidades motoras específicas. Estudo em jovens futebolistas e não futebolistas do sexo masculino dos 12 aos 16 anos de idade. Revista Portuguesa de Ciências do Desporto, 1(2), 22-35.

Vaeyens, R., Malina, R. M., Janssens, M., Van Renterghem, B., Bourgois, J., Vrijens, J., \& Philippaerts, R. M. (2006). A multidisciplinary selection model for youth soccer: the Ghent Youth Soccer Project. British Journal of Sports Medicine, 40(11), 928-934; discussion 934. http://doi.org/10.1136/bjsm.2006.029652

Valente-dos-Santos, J., Coelho-e-Silva, M. J., Simões, F., Figueiredo, A. J., Leite, N., Elferink-Gemser, M. T., ... Sherar, L. (2012). Modeling developmental changes in functional capacities and soccer-specific skills in male players aged 1117 years. Pediatric Exercise Science, 24(4), 603621.

Vandendriessche, J. B., Vaeyens, R., Vandorpe, B., Lenoir, M., Lefevre, J., \& Philippaerts, R. M.
(2012). Biological maturation, morphology, fitness, and motor coordination as part of a selection strategy in the search for international youth soccer players (age 15-16 years). Journal of Sports Sciences, 30(15), 1695-1703. http://doi.org/10.1080/02640414.2011.652654

Vänttinen, T., Blomqvist, M., Nyman, K., \& Häkkinen, K. (2011). Changes in body composition, hormonal status, and physical fitness in 11-, 13-, and 15-year-old Finnish regional youth soccer players during a two-year follow-up. Journal of Strength and Conditioning Research / National Strength \& Conditioning Association, 25(12), 3342-3351.

http://doi.org/10.1519/JSC.0b013e318236d0c2

Villar, R., \& Zuhl, C. A. (2006). Efeito da idade cronológica e da maturação biológica sobre a aptidão física em praticantes de futebol de 13 a 17 anos. Motricidade, 2(2), 69-79.

Weineck, J. (1999). Treinamento Ideal (9. ${ }^{a}$ ed.). São Paulo: MANOLE.

Williams, A. M., \& Franks, A. (1998). Talent identification in soccer. Sports, Exercise and Injury, 4, 159-165.

Williams, A. M., \& Reilly, T. (2000). Talent identification and development in soccer. Journal of Sports Sciences, 18(9), 657-667. http://doi.org/10.1080/02640410050120041 